\title{
Increased urinary CD80 excretion and podocyturia in Fabry disease
}

\author{
H. Trimarchi ${ }^{1 *}$, R. Canzonieri ${ }^{2}$, A. Schiel ${ }^{2}$, C. Costales-Collaguazo ${ }^{3}$, J. Politei $^{4}$, A. Stern ${ }^{2}$, M. Paulero $^{1}$, T. Rengel ${ }^{1}$, \\ J. Andrews ${ }^{1}$, M. Forrester ${ }^{1}$, M. Lombi ${ }^{1}$, V. Pomeranz ${ }^{1}$, R. Iriarte' ${ }^{1}$, A. Muryan ${ }^{2}$, E. Zotta ${ }^{3}$, M. D. Sanchez-Niño ${ }^{5,6^{*}+}$ \\ and A. Ortiz ${ }^{5,6+}$
}

\begin{abstract}
Background: Certain glomerulopathies are associated with increased levels of CD80 (B7-1). We measured the urinary excretion of CD80, podocyturia and proteinuria in controls and in subjects with Fabry disease either untreated or on enzyme replacement therapy (ERT).

Methods: Cross-sectional study including 65 individuals: controls $(n=20)$ and Fabry patients $(n=45,23$ of them not on ERT and 22 on ERT). Variables included age, gender, urinary protein/creatinine ratio (UPCR), estimated glomerular filtration rate (eGFR), urinary uCD80/creatinine ratio (uCD80) and podocyturia. CD80 mRNA expression in response to lyso-Gb3, a bioactive glycolipid accumulated in Fabry disease, was studied in cultured human podocytes.

Results: Controls and Fabry patients did not differ in age, eGFR and gender. However, UPCR, UCD80 and podocyturia were significantly higher in Fabry patients than in controls. As expected, Fabry patients not on ERT were younger and a higher percentage were females. Non-ERT Fabry patients had less advanced kidney disease than ERT Fabry patients: UPCR was lower and eGFR higher, but UCD80 and podocyturia did not differ between non-ERT or ERT Fabry patients. There was a significant correlation between UCD80 and UPCR in the whole population ( $r$ 0.44, p 0.0005) and in Fabry patients ( $\mathrm{r}$.42, p 0.0046). Lyso-Gb3 at concentrations found in the circulation of Fabry patients increased uCD80 expression in cultured podocytes.

Conclusions: Fabry disease is characterized by early occurrence of increased uCD80 excretion that appears to be a consequence of glycolipid accumulation. The potential for uCD80 excretion to reflect early, subclinical renal Fabry involvement should be further studied.
\end{abstract}

Keywords: Enzyme replacement therapy, Fabry disease, Lyso-Gb3, Podocyte, Podocyturia, CD80, Proteinuria

\section{Background}

Fabry disease is an $\mathrm{X}$-linked storage disease due to mutations in the GLA gene encoding $\alpha$-galactosidase A, leading to the lysosomal accumulation of enzyme substrates, namely globotriaosylceramide (Gb3), lysoglobotriaosylceramide (lyso-Gb3) and galabiosylceramide [1]. The intracellular pathological overload of these

\footnotetext{
*Correspondence: htrimarchi@hotmail.com; mdsanchez@fjd.es

†Sanchez-Niño MD and Ortiz A are contributed equally to this work

${ }^{1}$ Nephrology Service, Hospital Británico de Buenos Aires, Perdriel 74,

1280 Buenos Aires, Argentina

${ }^{5}$ IIS-Fundacion Jimenez Diaz, School of Medicine, UAM, Avda Reyes

Catolicos 2, 28040 Madrid, Spain

Full list of author information is available at the end of the article
}

glycophingolipids disturbs cell morphology and leads to cell dysfunction [2-4]. Gb3 is the best-known metabolite and accumulates mostly inside cells. However, in the extracellular space, Gb3 circulates inside lipoproteins and the concentration in Fabry males is only 2- to 4-fold above control values [5]. By contrast, lyso-Gb3, a more hydrosoluble derivative of Gb3, circulates at concentrations 200-to 500-fold above normal values [5]. The precise mechanisms by which these metabolites lead to cell dysfunction remain elusive. It has been speculated that the interaction of glycosphingolipids with ion channels or transporters, mainly localized within the endoplasmic reticulum, or the activation of inflammatory pathways may contribute to cell and tissue damage [6-8]. Thus, Gb3 levels correlated 
with oxidative stress and inflammation in Fabry patients $[9,10]$. Similar to diabetic nephropathy, Fabry nephropathy is a progressive proteinuric nephropathy of metabolic origin with a natural history that expands decades. In this regard, some glycolipids accumulated in Fabry disease, such as lyso-Gb3, are bioactive. Lyso-Gb3, but not Gb3, promoted the proliferation of vascular smooth muscle cells [11]. Moreover, lyso-Gb3 may activate inflammation pathways in renal cells [12]. Transforming growth factor- $\beta 1$ (TGF- $\beta 1$ ), Vascular endothelial growth factor (VEGF), Fibroblast growth factor-2 (FGF-2) - among others- are elevated in Fabry nephropathy, whilst Notch-1 signaling as well as apoptosis and autophagy-related inflammatory pathways are involved as well [13-16]. Specifically, at concentrations found in the circulation of Fabry patients, lyso-G3 promotes autocrine TGF- $\beta 1$ and Notch-1 signaling in podocytes, a response that mimics podocyte responses to high glucose concentrations [14, 17]. High glucose also increases CD80 expression in cultured podocytes [18]. Lymphocyte activation antigen 7-1, also known as CD80, is normally located on antigen presenting cells as neutrophils, macrophages and dendritic cells. CD80 modulates CD4+ and CD8+ T cell activity by interacting with the co-stimulator ligand CD28 or with cytotoxic T-lymphocyte protein 4 (CTLA-4) [19]. Normal podocytes do not express CD80. In certain glomerulopathies, including diabetic nephropathy, CD80 is expressed by podocytes and tubular cells and the renal excretion is increased $[18,20]$. Up-regulation of CD80 on podocytes and tubular cells under abnormal conditions suggests that these cells may behave as antigen-presenting cells and participate in inflammatory pathways [21]. Finally, CD80 has also been related to podocyte migration, detachment and podocyturia through its interaction with integrins. Podocyturia has been observed in active glomerulopathies [22, 23]. We have recently demonstrated the existence of podocyturia in Fabry disease, even in the absence of clinical nephropathy [24].

In this study, we explored the urinary excretion of CD80 in patients with Fabry disease and the potential drivers of CD80 expression.

\section{Methods}

This is cross-sectional, observational study included 65 individuals. A group of 20 healthy subjects without known clinical morbidities or pharmacological treatment was recruited among potential kidney donors and subjects with normal laboratory results and clinical history. In addition, 45 Fabry patients were studied. Of them 23 were not treated with enzyme replacement therapy (ERT) while 22 had received ERT for at least 12 months with agalsidase beta $1 \mathrm{mg} / \mathrm{kg}$ administered every fortnight (Fabrazyme, Genzyme Corp, Cambridge, MA,
USA). Fabry disease was diagnosed in all cases by low enzymatic alpha galactosidase A activity in dried blood spots and peripheral blood leukocytes, and confirmed by the identification of a GLA gene mutation. Patient characteristics are outlined in Table 1. The following variables were studied: age, gender, glomerular filtration rate estimated (eGFR) by the Chronic Kidney Disease-Epidemiology Collaboration equation (CKD-EPI), urinary protein-creatinine ratio (UPCR), podocyturia adjusted per gram of creatininuria and urinary CD80/creatinine ratio (uCD80).

\section{Podocyturia}

We have previously described the method to study podocyturia in detail [24]. Briefly, a mid-stream freshly voided urine sample was collected on-site after a minimum of $3 \mathrm{~h}$ without voiding; and $20 \mathrm{ml}$ of urine were centrifuged at $700 \mathrm{~g}$ for $5 \mathrm{~min}$ in a cytospin. The supernatant was discarded and the sediment was stored in $100 \mu \mathrm{l}$ aliquots at room temperature mixed with a $1.5 \mathrm{ml}$ solution of $40 \%$ formaldehyde diluted in phosphate-buffered saline (PBS) (pH 7.2-7.4) to reach a final $10 \%$ concentration. Podocyte nuclei were stained with 40,6-diamidino-2-phenylindole (DAPI). Podocytes were identified by immunofluorescence using anti-synaptopodin as the primary antibody (1:100, Abcam, Cambridge, MA, USA) and IgG anti-rabbit Alexa Fluor ${ }^{\circledR} 488$ (1:100, Abcam, Cambridge, MA, USA) as the secondary antibody. Samples were analyzed employing an epifluorescent microscope. Following our standardized technique, podocytes were counted in 10 randomly chosen $20 \times$ fields and the average of the counted podocytes in the microscopy fields was considered as the final count for each subject. Results were corrected based on the urinary creatinine concentration of the initial urinary volume of $20 \mathrm{ml}$ employed for podocyte counting [24, 25].

\section{CD80 and other determinations}

Serum creatinine was assessed the same week that urine was collected for podocyte counting. UPCR was measured from the specimen employed for podocyte assessment. The urinary concentration of CD80 was

$\begin{aligned} & \text { Table } 1 \text { General } \\
& \text { patients }\end{aligned}$
\begin{tabular}{lccc} 
Variables & Controls (n: 20) & Fabry (n: 45) & p value \\
\hline Age (years) & $30(20-48)$ & $33(11-86)$ & 0.92 \\
Gender (males) & $10(50 \%)$ & $18(40 \%)$ & 0.63 \\
eGFR (ml/min/1.73 m²) & $110(86-141)$ & $125(21-165)$ & 0.14 \\
UPCR (g/g) & $0.03(0.02-0.27)$ & $0.07(0.02-5.68)$ & 0.01 \\
Podocyturia/Cr (cells/g) & $4(0-90)$ & $32(0-439)$ & $<0.001$ \\
uCD80/Cr (ng/g) & $17(0-142)$ & $45(0-2966)$ & 0.04 \\
\hline
\end{tabular}

Significant values $(p<0.05)$ 
determined employing a Human CD80 Instant ELISA (Instant ELISA ${ }^{\circledR}$, affymetrix eBioscience, San Diego, CA USA).

\section{Ethics}

The present protocol was approved by the Institutional Review Board of the Hospital Británico de Buenos Aires, Buenos Aires, Argentina. Informed consent was obtained from each study participant.

\section{Cell culture and reagents}

Human podocytes are an immortalized cell line transfected with a temperature-sensitive SV40 gene construct and a gene encoding the catalytic domain of human telomerase $[14,17]$. At a permissive temperature of $33^{\circ} \mathrm{C}$, cells remain in an undifferentiated proliferative state and divide. Raising the temperature to $37{ }^{\circ} \mathrm{C}$ results in growth arrest and differentiation to the parental podocyte phenotype. Undifferentiated podocyte cultures were maintained at $33{ }^{\circ} \mathrm{C}$ in RPMI 1640 medium with penicillin, streptomycin, ITS (insulin, transferrin, selenite), and $10 \%$ FCS. Once cells reached 70-80\% confluence, they were fully differentiated by culture at $37{ }^{\circ} \mathrm{C}$ for at least 14 days $[14,17]$. Cells were cultured in serum-free media $24 \mathrm{~h}$ prior to addition of stimuli and throughout the experiment. Lyso-Gb3 (Sigma, St. Louis, MO) was used at a concentration of $100 \mathrm{nM}$ and tested negative for lipopolysaccharide. This concentration is clinically relevant, since circulating lyso-Gb3 has been reported to be in the 10-50 nM range for heterozygous females and above $100 \mathrm{nM}$ in males [11]. Lyso-Gb3 was chosen as a stimulus among the diverse glycolipids that accumulate in Fabry disease, because in relative terms its extracellular concentration is much higher (one order of magnitude higher) in Fabry patients than in controls than Gb3. Additionally, there is evidence that, unlike Gb3, lyso-Gb3 is bioactive in diverse cell systems, including vascular smooth muscle cells and podocytes [11, 14, 17]. In this regard, the $100 \mathrm{nM}$ concentration of lyso-Gb3 was previously shown to be bioactive in cultured human podocytes in dose response studies $[14,17]$.

\section{Real time reverse transcription-polymerase chain reaction}

RNA was isolated using Trizol reagent (Invitrogen, Paisley, UK). One microgram RNA was reverse transcribed with High Capacity cDNA Archive Kit (Applied Biosystems, Foster City, CA). Real-time PCR reactions were performed on the ABI Prism 7500 sequence detection PCR system (Applied Biosystems) according to the manufacturer's protocol using the DeltaDelta Ct method [14, 17]. Expression levels are given as ratios to GAPDH. Predeveloped primer and probe assays were from Applied Biosystems.

\section{Immunohistochemistry}

Kidney samples were obtained from excess tissue corresponding to kidney nephrectomy specimens donated to the biobank of the IIS-Fundacion Jimenez Diaz Biobank after diagnostic evaluation was performed. The local Ethics Committee approved the study protocol and informed consent was obtained. Control human kidney specimens were taken from normal portions of renal tissue from patients who underwent surgery because of localized renal tumors. One renal biopsy from a male Fabry patient was studied, a 69 year old with serum creatinine $4.2 \mathrm{mg} / \mathrm{dl}$, proteinuria $0.7 \mathrm{~g} / 24 \mathrm{~h}$. Immunohistochemistry was carried out in paraffin-embedded tissue section $5 \mu \mathrm{m}$ thick. The primary antibody was mouse monoclonal antiCD80 (1:100, Abcam). Sections were counterstained with Carazzi's hematoxylin. Negative controls included incubation with a non-specific immunoglobulin of the same isotype as the primary antibody.

\section{Statistical analysis}

Results are expressed as median and ranges. Variables were analyzed using the Wilcoxon Mann-Whitney test. Correlations between variables were obtained with the Spearman correlation coefficient. Results were considered significant when $\mathrm{p}<0.05$. The statistical program employed was InfoStat 2016, Córdoba Argentina.

\section{Results \\ Fabry patient studies}

Controls and Fabry patients did not diff in age, eGFR or gender. However, UPCR, uCD80 and podocyturia were significantly higher in Fabry patients (Table 1). Fabry patients not on ERT were younger and there was a higher proportion of females. This is expected because both younger patients and females usually have a milder disease and this may underlie the decision not to initiate ERT yet. In this regard, eGFR was higher and UPCR lower in patients not on ERT, probably reflecting milder kidney disease. However, there were no differences in uCD80 excretion or podocyturia between Fabry patients on ERT and not on ERT (Table 2). There was a significant correlation between uCD80 and UPCR in the whole population ( $\mathrm{r} 0.44, \mathrm{p} 0.0005)$ and in Fabry patients ( $\mathrm{r} 0.42$, p 0.0046). However, there was no correlation between uCD80 and podocyturia.

Since uCD80 was increased in the urine of Fabry patients, we next explored the expression of CD80 in human Fabry disease tissue. No expression of CD80 was observed in control kidneys. However, immunohistochemistry confirmed CD80 expression in podocytes in a kidney biopsy from a Fabry patient (Fig. 1). 
Table 2 Intragroup comparison of Fabry patients

\begin{tabular}{lllc}
\hline Variables & No ERT (n: 23) & ERT (n: 22) & p value \\
\hline Age (years) & $19(11-75)$ & $38.5(17-86)$ & 0.02 \\
Gender (males) & $4(14 \%)$ & $14(64 \%)$ & 0.01 \\
Time on ERT (months) & 0 & $42(34-50)$ & $<0.0001$ \\
eGFR (ml/min/1.73 m²) & $141(60-165)$ & $120.5(21-148)$ & 0.03 \\
UPCR (g/g) & $0.06(0.02-2.35)$ & $0.11(0.02-5.68)$ & 0.04 \\
Podocyturia/Cr (cells/g) & $34(4-439)$ & $24(0-107)$ & 0.45 \\
uCD80/Cr (ng/g) & $40(0-235)$ & $47(0-2966)$ & 0.7 \\
Mutations & D33G, L415P, R227X, A292T, N34D, C801, C326, C647A, & D33G, L415P, R227X, A292T, N34D, D264Y, D155H, L180F
\end{tabular}

Significant values $(p<0.05)$

Lyso-Gb3 increases CD80 expression in cultured podocytes Since uCD80 was increased early in Fabry nephropathy, even in the group of patients with better preserved eGFR and lower albuminuria, we explored whether glycolipids accumulated in Fabry disease may increase CD80 expression in kidney cells. We had previously shown that
lyso-Gb3, at concentrations found in the circulation of Fabry disease patients, increases the expression of diverse mediators of kidney injury in a time- and dose-dependent fashion, with peak response observed at $100 \mathrm{nM}$ and $24 \mathrm{~h}[14,17]$. Lyso-Gb3 at the concentration of $100 \mathrm{nM}$

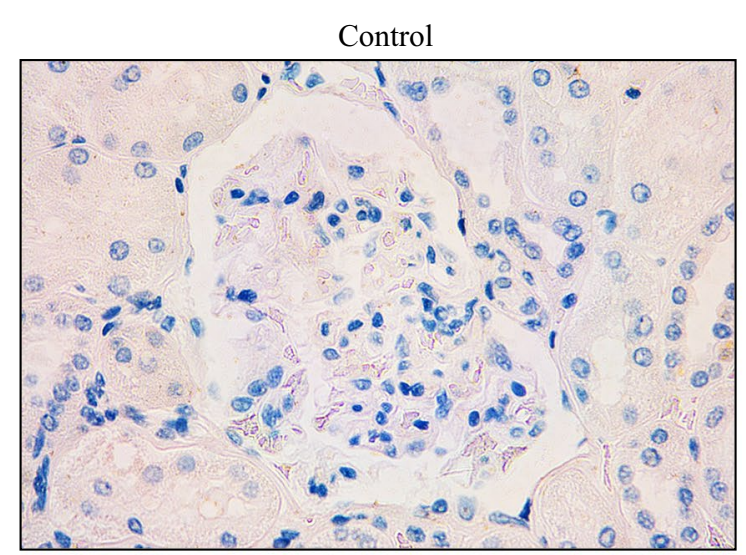

Fabry

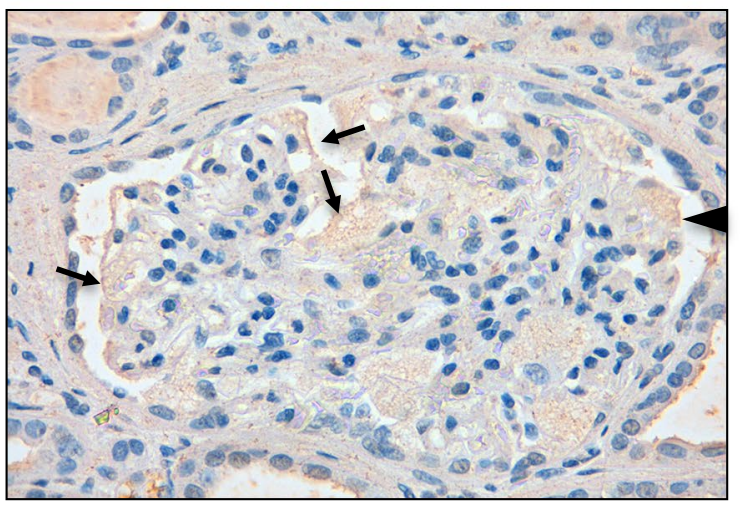

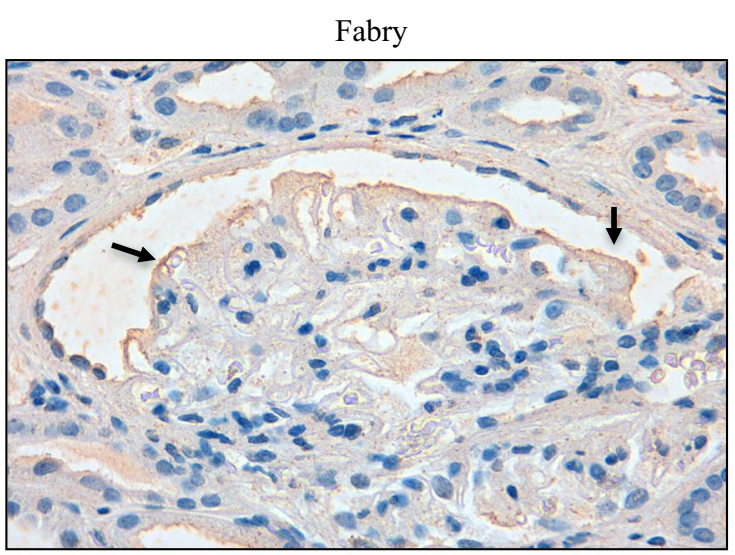

Fabry (Detail)

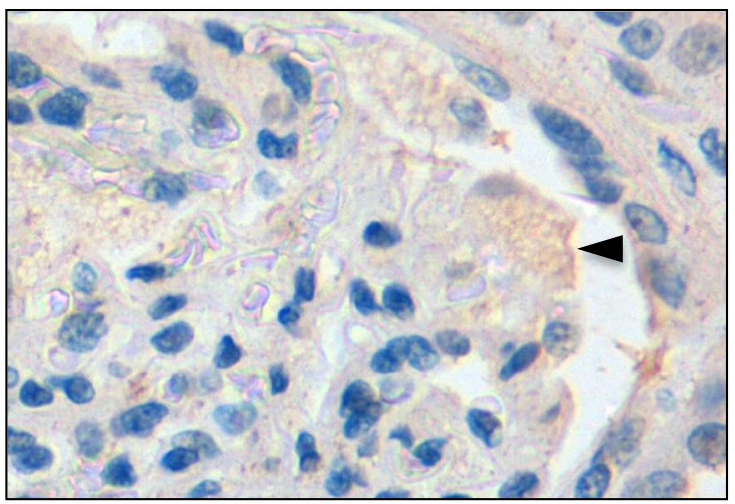

Fig. 1 Expression of CD80 in human Fabry disease. Immunohistochemistry of a human Fabry biopsy shows increased expression of CD80 in podocytes (arrows). Arrowhead indicates the vacuolated, enlarged and CD80 stained podocyte observed in the detail. No staining was found in human control biopsies. Original magnification $\times 20$ and $\times 40$ 
induced an increase in the mRNA expression of CD80 that peaked at $24 \mathrm{~h}$ in podocytes (Fig. 2).

\section{Discussion}

In the present study, we have confirmed that in Fabry disease podocytes are lost in urine in higher amounts than in controls and that this is an early feature, present already in patients with subclinical kidney disease. If maintained over time, this may lead to podocyte loss and to the classical glomerular lesion of Fabry nephropathy: focal and segmental glomerulosclerosis. A novel finding is that uCD80 excretion is also increased early in Fabry nephropathy. While there was a correlation between $\mathrm{uCD} 80$ and proteinuria, the lack of correlation between uCD80 and podocyturia suggests that podocyturia appears not to be linked to the activation of CD80 signaling pathways and that $\mathrm{uCD} 80$ and podocyturia reflect different aspects of kidney injury in Fabry nephropathy. Moreover, we have identified lyso-Gb3 as a potential driver of CD80 expression in podocytes. Lyso-Gb3 had been previously shown to increase the expression or secretion of TGF $\beta 1$, CD74, Notch1 and MCP-1 in podocytes $[14,17]$. Thus, lyso-Gb3 appears to promote a generalized stress response that we have now shown to also include CD80 and that is reminiscent of high glucoseelicited podocyte responses [18, 26, 27]. In this regard, another glycolipid, bacterial lipopolysaccharide, also increases CD80 expression in podocytes [28].

In Fabry disease, silent and heavier podocyturia occurred in younger individuals when median eGFR and proteinuria were still normal. Furthermore, uCD80 was already elevated in younger patients and females that

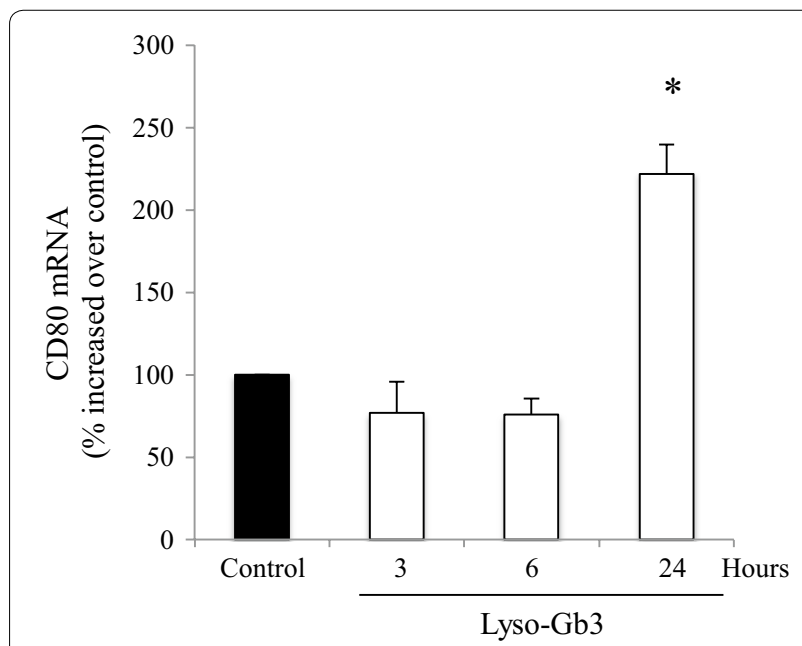

Fig. 2 Lyso-Gb3 upregulates CD80 mRNA expression in podocytes. Cultured human podocytes were stimulated with 100 nM lyso-Gb3. Time-course of CD80 mRNA induction. * $p<0.004$ vs control. Expression of mRNA was assessed by real time RT-PCR. Mean \pm SEM of three independent experiments were not yet on ERT, suggesting that it may be an early marker of kidney injury. In addition, uCD80 remained increased in patients on ERT. Since this was a crosssectional study, it is unknown whether uCD80 had been higher at the start of ERT and had decreased but not normalized with ERT. Whether earlier therapeutic interventions could normalize uCD80 levels requires further investigations. The current literature remarks that in Fabry disease, early ERT initiation is associated to better long-term results $[29,30]$. However, our cell culture data are consistent with the uCD80 observation, since lysoGb3 is decreased but usually not normalized by ERT [11].

Fabry nephropathy is a glomerulopathy. Thus, it is not surprising that the metabolic defect triggers the expression of inflammatory and fibrotic molecules, growth factors, cytokines and chemokines, as is the case for other glomerulopathies [31,32]. In addition, a characteristic of Fabry nephropathy is that besides podocytes, endothelial and tubular cells accumulate glycolipids. These cells are all potential sources of inflammatory mediators. However, we were unable to measure simultaneously CD80 in blood and in urine and, thus, cannot determine whether uCD80 is of kidney origin. Notwithstanding these observations, our finding underscores the concept that in Fabry nephropathy, inflammatory molecules are excreted in urine and this appears not to be normalized by ERT. CD80 is not exclusive to lymphocytes, as podocytes in primary focal and segmental glomerulosclerosis [33] and diabetes [18] and podocytes and tubular cells in IgA nephropathy express CD80 in human biopsies [20]. Although some authors have remarked a potential role of CD80 in certain glomerulopathies, such as IgA nephropathy, primary focal and segmental glomerulonephritis and crescentic glomerulopathies [20, 21, 33, 34], others have recently seriously questioned these findings, particularly in minimal change disease and primary focal and segmental glomerulosclerosis [35]. Recent data point to glycogen synthase kinase (GSK) 3 as a key player in the pathogenesis of podocytopathy and proteinuria. In GSK3 $\beta$ knockout mice, de novo podocyte expression of nuclear factor kappa B (NFKB)-dependent mediators of podocyte injury, including $C D 80$, cathepsin $\mathrm{L}$, and MCP1 , was reduced [36]. These molecules may contribute to the pathophysiology of Fabry disease [14, 16, 37]. In Fabry disease, kidney cell CD80 expression may be driven by accumulated glycolipids, as shown in our cell culture experiments, and thus, it may provide information about continuing kidney injury by glycolipids despite ERT. Additional cytokines in the local microenvironment may also contribute.

It is currently unknown whether podocyte CD80 expression contributes to the pathogenesis of Fabry nephropathy by altering the shape, rearranging actin and 
altering glomerular permeability thus promoting proteinuria [21, 34]. Indeed, CD80 has been implicated in podocyte contraction, migration and eventual podocyte loss $[21,34]$. However, we did not find a correlation between uCD80 and podocyturia. This may be related to small sample size. Alternatively, uCD80 and podocyturia may represent different aspects of Fabry nephropathy. Abnormal podocyte CD80 up-regulation has been linked to $\beta 1$ integrin subunit abnormalities $[21,34,38]$. In this regard, increased urinary excretion of $\beta 3$ integrin was increased in Fabry patients [38].

Since upregulated podocyte CD80 expression has been observed in several glomerulopathies, increased uCD80 levels are not expected to be diagnostic of Fabry nephropathy. Thus, they cannot presently replace renal biopsy. At present, assessment of podocyturia should be considered an experimental procedure. While it is a promising technique, since podocyte turnover is very limited and loss of podocytes results in irreversible glomerular injury, methods are not standardized for routine clinical assessment. It is likely that podocyturia is influenced by different factors, including availability of podocytes (it may decrease with advanced kidney disease due to loss of glomeruli) and rate of injury or death of remaining podocytes. In this regard, molecules expressed in stressed podocytes are likely shed and be present in urine as a result, without necessarily implying the urinary excretion of the podocyte of origin.

There are several limitations in our study: In absolute terms, the number of patients is small and the study is cross-sectional. However, few studies in rare renal diseases are able to recruit higher number of patients, and a prospective study will require several years of followup, given the 40 year natural history of Fabry nephropathy. We have not directly addressed the cellular source of CD80 in vivo in the studied patients. Whether the uCD80 originates from lymphocytes, neutrophils and/ or dendritic cells, or from abnormal CD80 expression in podocytes, tubular or endothelial cells is unknown. Our findings are based on a highly sensitive and specific human ELISA, but are limited to urine, and we cannot discern whether there are differences in the behavior of circulating and urinary CD80 levels. However, an unrelated Fabry kidney biopsy and cell culture data suggest that podocytes could be one of the sources of uCD80. While some patients were off ERT and others on ERT, the cross sectional nature of the study does not allow to draw definitive conclusions on the role of podocyturia and excretion of CD80 as treatment monitoring biomarkers. The off ERT and on ERT groups are not comparable. Patients not yet on ERT were significantly younger and we have only one assessment for patients on ERT: in the absence of baseline values, it is unknown how these parameters might have changed upon initiation of ERT.

In summary, we confirmed the early development of podocyturia in Fabry patients. Moreover, we observed an early increase in $\mathrm{uCD} 80$. The fact that CD80 expression is increased by lyso-Gb3 in podocytes suggests that $\mathrm{UCD} 80$ may be an early marker of kidney injury in Fabry disease and also a marker of continued renal injury by glycolipids and future studies exploring the potential of uCD80 to reflect early, subclinical renal Fabry involvement are warranted.

\section{Conclusions}

In Fabry disease podocyturia and urinary excretion of CD80 rise early in the disease process, even in patients with normal renal function. CD80 expression appears to be driven by exposure to glycolipids such as lyso-Gb3. This suggests that podocyturia and urinary CD80 expression may provide information into early and continuing kidney injury in Fabry disease. This hypothesis should be confirmed in larger longitudinal studies.

\section{Abbreviations}

CD74: cluster of differentiation 74; CD80 (B7-1): lymphocyte activation antigen 7-1; CKD-EPI: chronic kidney disease-epidemiology collaboration; CTLA-4: cytotoxic T-lymphocyte protein 4; DAPI: 40,6-diamidino-2-phenylindole; eGFR: estimated glomerular filtration rate; ERT: enzyme replacement therapy; FGF-2: fibroblast growth factor-2; GAPDH: glyceraldehyde 3-phosphate dehydrogenase; Gb3: globotriaosylceramide; GLA: galactosidase alpha; GSK 3: glycogen synthase kinase; ITS: insulin, transferrin, selenite; Lyso-Gb3: lyso-globotriaosylceramide; MCP1: monocyte chemoattractant protein-1; NFkB: nuclear factor kappa B; PBS: phosphate-buffered saline; PCR: polymerase chain reaction; RNA: ribonucleic acid; TGF- $\beta$ 1: transforming growth factor- $\beta 1$; UPCR: urinary protein/creatinine ratio; VEGF: vascular endothelial growth factor.

\section{Authors' contributions}

MDSN performed the cell culture and histological studies. HT, MDSN and AO wrote the manuscript. RC, AS, CCC, JP, AS, MP, TR and JA collected clinical data and urine samples. MF, ML, VP, RI, AM and EZ studied podocyturia and urinary CD80 expression. All authors read and approved the final manuscript.

\section{Author details \\ ${ }^{1}$ Nephrology Service, Hospital Británico de Buenos Aires, Perdriel 74 1280 Buenos Aires, Argentina. ${ }^{2}$ Central Laboratory, Hospital Británico de Buenos Aires, Buenos Aires, Argentina. ${ }^{3}$ IFIBIO Houssay, CONICET, Physiopa- thology, Pharmacy and Biochemistry Faculty, Universidad de Buenos Aires, Buenos Aires, Argentina. ${ }^{4}$ Neurology Department, Laboratorio Neuroquímica Dr. Néstor Chamoles, Buenos Aires, Argentina. ${ }^{5}$ IIS-Fundacion Jimenez Diaz, School of Medicine, UAM, Avda Reyes Catolicos 2, 28040 Madrid, Spain. ${ }^{6}$ RED- INREN, Madrid, Spain.}

\section{Acknowledgements \\ We want to thank María Laura Ares and Marina Fernandez for their profes- sional assistance.}

\section{Availability of data and materials}

Available data are presented in the manuscript. For any additional information, please contact the corresponding author.

\section{Consent for publication}

No individual person data are presented. 


\section{Disclosures}

Hernan Trimarchi is consultant to Genzyme for the product Fabrazyme and to Brystol Myers Squibb for the product belatacept. Alberto Ortiz and Juan Politei are consultant to Genzyme for the product Fabrazyme. Alberto Ortiz has received speaker fees from Genzyme and Shire and Maria Dolores SanchezNiño from Genzyme.

\section{Ethics approval and consent to participate}

The present protocol was approved by the Institutional Review Board of the Hospital Británico de Buenos Aires, Buenos Aires, Argentina. Informed consent was obtained from each study participant. Kidney samples were obtained from excess tissue corresponding to kidney nephrectomy specimens donated to the biobank of the IIS-Fundacion Jimenez Diaz Biobank after diagnostic evaluation was performed. The local Ethics Committee approved the study protocol and informed consent was obtained for kidney tissue obtained after 2007. In 2012 the local Ethics Committee authorized the transfer to the biobank of all kidney tissue obtained for diagnostic purposes before 2007 that remained unused in 2012 and waived the need to request consent for this transfer.

\section{Funding}

This work was supported by FIS PI13/00047, CP14/00133, PI15/00298,

PI16/02057, FEDER funds ISCIII-RETIC REDinREN RD12/0021, IBERERC, Sociedad Española de Nefrología, Programa Intensificacion Actividad Investigadora (ISCIII/Agencia Lain-Entralgo/CM) to AO, Miguel Servet MS14/00133 to MDSN. This work has been supported by the "VI Convocatoria de ayudas investigación sobre medicamentos huérfanos y enfermedades raras con la ayuda Fundación Cajasol".

Received: 10 August 2016 Accepted: 3 October 2016

\section{Published online: 13 October 2016}

\section{References}

1. Desnick RJ, loannou Y, Eng CM. a-Galactosidase A deficiency: Fabry disease. In: Scriver CR, Beaudet AL, Sly WS, Valle D, editors. The metabolic and molecular bases of inherited disease. New York: McGraw-Hill; 2001. p. 3733-74

2. Askari H, Kaneski CR, Semino-Mora C, Desai P, Ang A, Kleiner DE, et al. Cellular and tissue localization of globotriaosylceramide in Fabry disease. Virchows Arch. 2007;451:823-34.

3. Merscher S, Fornoni A. Podocyte pathology and nephropathy — sphingolipids in glomerular diseases. Front. Endocrinol. (Lausanne). 2014; 5:127.

4. Swiatecka-Urban A. Membrane trafficking in podocyte health and disease. Pediatr Nephrol. 2013;28:1723-37.

5. Rombach SM, Dekker N, Bouwman MG, Linthorst GE, Zwinderman AH, Wijburg FA, et al. Plasma globotriaosylsphingosine: diagnostic value and relation to clinical manifestations of Fabry disease. Biochim Biophys Acta. 2010;1802:741-8

6. Altarescu G, Moore DF, Pursley R, Campia U, Goldstein S, Bryant M, et al. Enhanced endothelium-dependent vasodilation in Fabry disease. Stroke. 2001:32:1559-62.

7. Lloyd-Evans E, Pelled D, Riebeling C, Bodennec J, de-Morgan A, Waller $\mathrm{H}$, et al. Glucosylceramide and glucosylsphingosine modulate calcium mobilization from brain microsomes via different mechanisms. J Biol Chem. 2003:278:23594-9.

8. Pelled D, Lloyd-Evans E, Riebeling C, Jeyakumar M, Platt FM, Futerman $\mathrm{AH}$. Inhibition of calcium uptake via the sarco/endoplasmic reticulum Ca2 + -ATPase in a mouse model of Sandhoff disease and prevention by treatment with N-butyldeoxynojirimycin. J Biol Chem. 2003;278:29496-501.

9. Biancini GB, Jacques CE, Hammerschmidt T, de Souza HM, Donida B, Deon $\mathrm{M}$, et al. Biomolecules damage and redox status abnormalities in Fabry patients before and during enzyme replacement therapy. Clin Chim Acta. 2016:461:41-6.

10. Biancini GB, Vanzin CS, Rodrigues DB, Deon M, Ribas GS, Barschak AG, et al. Globotriaosylceramide is correlated with oxidative stress and inflammation in Fabry patients treated with enzyme replacement therapy. Biochim Biophys Acta. 2012;1822:226-32.
11. Aerts JM, Groener JE, Kuiper S, Donker-Koopman WE, Strijland A, Ottenhoff $\mathrm{R}$, et al. Elevated globotriaosylsphingosine is a hallmark of Fabry disease. Proc Natl Acad Sci USA. 2008;105:2812-7.

12. Trimarchi H. The kidney in Fabry disease. More than mere sphyngolipids overload. J Inborn Errors Metab Screen. 2016;4:1-5.

13. Lee $\mathrm{MH}, \mathrm{Choi} \mathrm{EN}$, Jeon $\mathrm{YJ}$, Jung S-C. Possible role of transforming growth factor- $\beta 1$ and vascular endothelial growth factor in Fabry disease nephropathy. Int J Mol Med. 2012;30:1275-80.

14. Sanchez-Niño MD, Carpio D, Sanz AB, Ruiz-Ortega M, Mezzano S, Ortiz A. Lyso-Gb3 activates Notch1 in human podocytes. Hum Mol Genet. 2015:24:5720-32.

15. Liebau MC, Braun F, Höpker K, Weitbrecht C, Bartels V, Müller R-U, et al. Dysregulated autophagy contributes to podocyte damage in Fabry's disease. PLoS One. 2013;8:e63506.

16. Trimarchi H, Karl A, Raña MS, Forrester M, Pomeranz V, Lombi F, et al. Initially nondiagnosed Fabry's disease when electron microscopy is lacking: the continuing story of focal and segmental glomerulosclerosis. Case Rep Nephrol Urol. 2013:3:51-7.

17. Sanchez-Niño MD, Sanz AB, Carrasco S, Saleem MA, Mathieson PW, Valdivielso JM, et al. Globotriaosylsphingosine actions on human glomerular podocytes: implications for Fabry nephropathy. Nephrol Dial Transplant. 2011;26:1797-802.

18. Fiorina P, Vergani A, Bassi R, Niewczas MA, Altintas MM, Pezzolesi MG, et al. Role of podocyte B7-1 in diabetic nephropathy. J Am Soc Nephrol. 2014;25:1415-29.

19. Linsley PS, Ledbetter JA. The role of the CD28 receptor during T cell responses to antigen. Annu Rev Immunol. 1993;11:191-212.

20. Wu Q, Jinde K, Endoh M, Sakai H. Clinical significance of costimulatory molecules CD80/CD86 expression in IgA nephropathy. Kidney Int. 2004;65:888-96.

21. Trimarchi H. Abatacept and glomerular diseases: the open road for the second signal as a new target is settled down. Recent Pat Endocr Metab Immune Drug Discov. 2015:9:2-14.

22. Hara M, Yanagihara T, Kihara I. Cumulative excretion of urinary podocytes reflects disease progression in IgA nephropathy and Schönlein-Henoch purpura nephritis. Clin J Am Soc Nephrol. 2007;2:231-8.

23. Yu D, Petermann A, Kunter U, Rong S, Shankland SJ, Floege J. Urinary podocyte loss is a more specific marker of ongoing glomerular damage than proteinuria. J Am Soc Nephrol. 2005;16:1733-41.

24. Trimarchi H, Canzonieri R, Schiel A, Politei J, Stern A, Andrews J, et al. Podocyturia is significantly elevated in untreated vs treated Fabry adult patients. J Nephrol. 2016. doi:10.1007/s40620-016-0271-z.

25. Sabino AR, Teixeira VP, Nishida SK, Sass N, Mansur JB, Kirsztajn GM. Detection of podocyturia in patients with lupus nephritis. J Bras Nefrol. 2013;35:252-8.

26. Sanchez-Niño M-D, Bozic M, Córdoba-Lanús E, Valcheva P, Gracia O, Ibarz $M$, et al. Beyond proteinuria: VDR activation reduces renal inflammation in experimental diabetic nephropathy. Am J Physiol Renal Physiol. 2012:302:F647-57.

27. Sanchez-Niño MD, Sanz AB, Ihalmo P, Lassila M, Holthofer H, Mezzano $\mathrm{S}$, et al. The MIF receptor CD74 in diabetic podocyte injury. J Am Soc Nephrol. 2009;20:353-62.

28. Reiser J, von Gersdorff G, Loos M, Oh J, Asanuma K, Giardino L, et al. Induction of B7-1 in podocytes is associated with nephrotic syndrome. J Clin Invest. 2004:113:1390-7.

29. Ito S, Ogura M, Kamei K, Matsuoka K, Warnock DG. Significant improvement in Fabry disease podocytopathy after 3 years of treatment with agalsidase beta. Pediatr Nephrol. 2016;31:1369-73.

30. Tøndel C, Bostad L, Larsen KK, Hirth A, Vikse BE, Houge G, et al. Agalsidase benefits renal histology in young patients with Fabry disease. J Am Soc Nephrol. 2013;24:137-48.

31. Suzuki H, Kiryluk K, Novak J, Moldoveanu Z, Herr AB, Renfrow MB, et al. The pathophysiology of IgA nephropathy. J Am Soc Nephrol. 2011;22:1795-803.

32. Trimarchi H. Primary focal and segmental glomerulosclerosis. Why are there pieces still hidden in this puzzle? Eur Med J Nephrol. 2015:3:104-10.

33. Yu C-C, Fornoni A, Weins A, Hakroush S, Maiguel D, Sageshima J, et al. Abatacept in B7-1-positive proteinuric kidney disease. N Engl J Med. 2013;369:2416-23.

34. Reiser J, Alachkar N. Proteinuria: abate or applaud abatacept in proteinuric kidney disease? Nat Rev Nephrol. 2014;10:128-30. 
35. Novelli R, Gagliardini E, Ruggiero B, Benigni A, Remuzzi G. Any value of podocyte B7-1 as a biomarker in human MCD and FSGS? Am J Physiol Renal Physiol. 2016;310:F335-41.

36. Li C, Ge Y, Dworkin L, Peng A, Gong R. The $\beta$ isoform of GSK3 mediates podocyte autonomous injury in proteinuric glomerulopathy. J Pathol. 2016;239:23-35.
37. Chen K-H, Chien Y, Wang K-L, Leu H-B, Hsiao C-Y, Lai Y-H, et al. Evaluation of proinflammatory prognostic biomarkers for fabry cardiomyopathy with enzyme replacement therapy. Can J Cardiol. 2015. doi:10.1016/j. cjca.2015.10.033.

38. Utsumi K, Itoh K, Kase R, Shimmoto M, Yamamoto N, Katagiri Y, et al. Urinary excretion of the vitronectin receptor (integrin alpha $V$ beta 3 ) in patients with Fabry disease. Clin Chim Acta. 1999;279:55-68.

\section{Submit your next manuscript to BioMed Central and we will help you at every step:}

- We accept pre-submission inquiries

- Our selector tool helps you to find the most relevant journal

- We provide round the clock customer support

- Convenient online submission

- Thorough peer review

- Inclusion in PubMed and all major indexing services

- Maximum visibility for your research

Submit your manuscript at www.biomedcentral.com/submit 